\title{
Studies in alcoholic liver disease in Britain
}

\section{Clinical and pathological patterns related to natural history}

\author{
P. W. BRUNT ${ }^{1}$, M. C. KEW, P. J. SCHEUER, AND SHEILA SHERLOCK \\ From the Departments of Medicine and Morbid Anatomy, Royal Free Hospital Medical School, London
}

SUMMARY A group of 258 patients with various forms of alcoholic liver disease-steatosis, mild and severe hepatitis, and cirrhosis-has been studied. Severity of disease as judged histologically did not correlate very well with clinical presentation although signs of hepatocellular failure were certainly commoner in severe hepatitis and cirrhosis. Fever, pigmentation, and clubbing were also pointers to these two conditions. Alcoholic hepatitis is probably precirrhotic and carries a poor prognosis and the best laboratory indicators of this are moderate elevation of white cell count and bilirubin. Prognosis in alcoholic liver disease is significantly improved by abstinence from alcohol.

Alcoholic liver disease is a major cause of ill health and mortality. In the United States cirrhosis, most of it alcoholic, is now the fourth commonest cause of death in males in the fourth to sixth decades (Popper, Davidson, Leevy, and Schaffner, 1969). In Britain it is certainly less frequent but its true extent is difficult to estimate. Evidence suggests that alcoholism as a social and medical disease (and presumably also alcoholic liver disease) having decreased in frequency during the earliest years of the century is onceagain on the increase (Office of Health Economics, 1970). In contrast to a wide study of this problem in the United States and Europe remarkably little is known about the nature and outcome of alcoholic liver disease in this country. The present survey was carried out to study the clinical and histopathological forms of the disease, to relate these forms to the outcome, and to define factors which may influence prognosis.

\section{Patients and Methods}

Records of the Medical Professorial Unit of the Royal Free Hospital were surveyed for all cases of alcoholic liver disease in the years 1960 to 1967 , a total of 258 patients. Up-to-date information on state of health, drinking habits, and liver function was sought from the patients' practitioners and, where appropriate, patients were recalled for review.

'Correspondence to Dr P. W. Brunt, Department of Medicine, Medical School, Foresterhill, Aberdeen, AB9 2ZB.

Received for publication 8 October 1973
In the case of death hospital records and postmortem reports were obtained. Histological specimens (190) from 132 patients were reviewed by one of us (P.J.S.) working independently and without access to clinical data.

Criteria for diagnosing 'liver disease due to alcohol' are not easy to establish but in all cases retained there was a clear history of alcohol intake of 'heavy social proportions' or more. A few cases were rejected where alcohol intake appeared to be occasional or indefinite or where clinical and histological features strongly suggested another well defined disease pattern.

\section{Results}

Of the 258 patients, $183(71 \%)$ were males and 75 $(29 \%)$ were females. There was no sex difference in the age distribution being a mean of 50.0 years (range 32-70) and 50.4 (range 31-72) respectively. Patients were assigned to two groupings according to drinking patterns and histological classification.

\section{ALCOHOL CONSUMPTION PATTERNS}

Every effort was made to try and obtain an accurate assessment of patients' intake by using information from patients themselves, from relatives, and from family doctors. It is appreciated that in most cases this could only be approximate, the difficulties of obtaining the truth from alcoholics being notorious. In most cases it was possible to assign patients to one of three groups: group 1, 'moderate', that is heavy drinking by social standards, but appreciably less 
than in groups 2 and 3 ; group 2, 'intermittent heavy', heavy drinking, usually in excess of half a bottle of spirits a day or its equivalent ( $120 \mathrm{~g}$ of ethanol), but with evidence of some periods of abstinence or very limited intake; group 3, 'continuous heavy', continuous heavy drinking with few or no periods of abstinence, frequently with evidence of addiction, daily intake usually in excess of half a bottle of spirits or equivalent ( $120 \mathrm{~g}$ of ethanol). Most of the patients fell into groups 2 and $3(49 \%$ and $41 \%$ respectively), leaving only $10 \%$ in group 1 . There was no sex difference in these categories. While drinking patterns did not seem materially to influence the type of liver disease produced (see below) they did influence the outcome in that $33 \%$ of group 3 patients and $29 \%$ of group 2 patients died during the period of review as compared with only $21 \%$ of group 1. There was no difference in mode of death according to groups.

Spirit drinking accounted for the major part of the series $-55 \%$ of all patients. Only $9 \%$ were pure beer drinkers and $2.0 \%$ pure wine drinkers. The remainder $(34 \%)$ had mixed drinking patterns, predominantly spirits and beer. Long duration of drinking was a feature of most patients, nearly nine out of $\mathbf{1 0}$ claiming to have drunk heavily for more than 10 years and only $3.5 \%$ claiming less than five years. There was no apparent association between duration and the histological type.

\section{HISTOPATHOLOGY}

The frequency of histological patterns in the first or only specimen seen is shown in table I. Ten per cent of first specimens were normal or showed fatty liver alone. The same number showed mild alcoholic hepatitis together with fatty change, or fatty change and scarring. More severe alcoholic hepatitis but without cirrhosis was found in 15 patients $(11 \%)$, and the same number of biopsies showed fibrosis without alcoholic hepatitis. Alcoholic hepatitis was

\begin{tabular}{lll}
\hline Histological Characteristic & No. of Patients & \\
\hline Fatty change & 100 & $(76 \%)$ \\
Scarring (non-cirrhotic) & 41 & $(31 \%)$ \\
Alcoholic hepatitis (all) & 70 & $(53 \%)$ \\
Without cirrhosis & 29 & $(22 \%)$ \\
With cirrhosis & 41 & $(31 \%)$ \\
Cirrhosis (all) & 75 & $(57 \%)$ \\
With hepatitis & 41 & $(31 \%)$ \\
Without hepatitis & 34 & $(26 \%)$ \\
Micronodular & 47 & $(0 \cdot 8 \%)$ \\
Macronodular & 1 & $(10 \%)$ \\
Mixed type & 13 & $(11 \%)$ \\
Undetermined type & 14 &
\end{tabular}

Table I Frequency of individual histological characteristics in first (or only) specimens (needle or wedge biopsy or necropsy) defined by focal, usually centrolobular liver cell swelling and necrosis, and infiltration by neutrophils. Fourteen specimens with a typical inflammatory lesion of alcoholic hepatitis had no detectable Mallory's hyaline, and scanty hyaline was found in the absence of the inflammatory lesion in a further 13, which were not coded as alcoholic hepatitis.

The commonest single pattern of the first biopsy was cirrhosis with both fatty 'change and severe alcoholic hepatitis $(18 \%)$, followed by fatty cirrhosis without hepatitis $(12 \%)$ and severe hepatitis with scarring but no evidence of cirrhosis ( $11 \%)$.

The cirrhosis appeared micronodular in two-thirds of all the cirrhotic samples, and mixed or macronodular in most of the remainder. It is recognized, however, that determination of the anatomical type of cirrhosis in needle biopsies cannot always be made accurately because of possible sampling error. In over half the cirrhotic biopsies cirrhosis appeared fully developed, while in the others it was early, suspected, or the stage of development was not determined. Cholestasis was uncommon and usually slight.

Repeat specimens were available in 53 patients. In 27 of these there was a change in pattern, such as the appearance of cirrhosis following alcoholic hepatitis, or disappearance of an inflammatory or necrotic element. Of 12 patients whose initial biopsies showed hepatitis (without cirrhosis), seven went on to develop cirrhosis in the second biopsy whereas four still showed hepatitis (one only reverting to fatty change). By contrast, of seven patients showing no evidence of either hepatitis or cirrhosis on the initial biopsy, six were almost certainly non-cirrhotic and the seventh probably non-cirrhotic on the second biopsy. The range of time between first and second biopsies in the series was four months to seven years. Only one of the seven patients with progression from hepatitis to cirrhosis was known to become abstinent during the period of study. Primary liver cell carcinoma was seen at necropsy in two patients, whose first specimens had shown cirrhosis without fat or alcoholic hepatitis.

Stainable iron was found in liver cells in $63(39 \%)$ of 161 specimens where a section stained for iron was available. Forty-seven of these showed grade 1 iron (0-4 grading system), 13 grade 2, and three grade 3. In none was grade 4 siderosis found. A further four specimens showed iron in reticuloendothelial cells only. More than half the siderotic specimens werefrom cirrhotic livers.

CORRELATION OF CLINICAL AND

HISTOLOGICAL FEATURES

Patients were assigned to one of five categories: (1) fatty liver, (2) mild alcoholic hepatitis, (3) severe 
alcoholic hepatitis, (4) cirrhosis without alcoholic hepatitis, and (5) cirrhosis with alcoholic hepatitis.

There was no apparent relation of age to type of disease but there was a sex relationship (table II) in that there were more females with hepatitis $(2+3)$ by comparison with cirrhosis $(4+5)$ and fatty liver (1).

The distribution of drinking groups 1 to 3 within the types of disease did not differ significantly. There was a slight excess of group 3 in severe hepatitis (c) which was not statistically significant (table III). An 'intake score' was calculated for each histological type, being the arithmetic mean of all the numbers 1 to 3 inversely assigned to each patient individually according to his drinking pattern grouping. This is an approximate guide to the overall drinking habits of that particular histological group. Again there was no significant difference between the types although the heaviest drinkers do appear in the severe hepatitis group.

Modes of presentation are shown in figure 1. Non-specific symptoms consisted of anorexia, nausea, vomiting, weight loss, and abdominal pain. Approximately one quarter of the patients were first noted to have hepatomegaly, often during routine medical check-up or when seen for some unrelated condition. Features suggesting hepatocellular failure-jaundice and fluid retention were much less common, accounting for about one patient in 10 over the whole series, and occurring mainly in those with severe hepatitis or cirrhosis. In only the

\begin{tabular}{llll}
\hline Histological Typing & Male (\%) & Female (\%) \\
\hline Fatty liver & 79 & 21 \\
Hepatitis (mild and severe) & 59 & 41 \\
Cirrhosis with or without & 72 & 28 \\
hepatitis & 183 & 75 \\
Total number in series & &
\end{tabular}

Table II Sex distribution according to histological typing for entire series

'severe hepatitis' group did encephalopathy predominate in the presenting picture. Haemorrhage (from varices and ulceration) was predictably morecommon in the cirrhotics.

Associated clinical phenomena are shown ine figures 2 and 3. Pancreatitis was seen in only $6 \%$ of

\begin{tabular}{lllll}
\hline Histological Subtype & $\begin{array}{l}\text { Intake } \\
\text { Score }\end{array}$ & $\begin{array}{l}\text { Group 1: } \\
\text { Moderate } \\
(\%)\end{array}$ & $\begin{array}{l}\text { Group 2: } \\
\text { Intermit- } \\
\text { tent } \\
\text { Heavy } \\
(\%)\end{array}$ & $\begin{array}{l}\text { Group 3: } \\
\text { Continuous } \\
\text { Heavy } \\
(\%)\end{array}$ \\
\hline Fatty liver & 1.77 & 8 & 60 & 32 \\
Mild hepatitis & 1.63 & 8 & 46 & 46 \\
Severe hepatitis & 1.47 & 6 & 35 & 65 \\
Cirrhosis & 1.77 & 13 & 45 & 42 \\
Cirrhosis \pm & 1.59 & 5 & 49 & 46 \\
hepatitis & & 10.5 & 49 & 40.5 \\
All groups & & & & \\
\hline
\end{tabular}

Table III Histological subtypes related to drinking patterns
GROUP 1 FAT

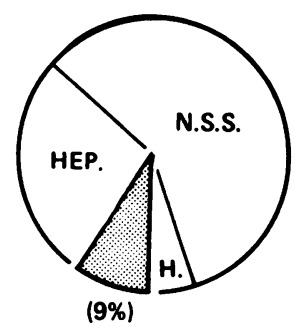

GROUP 4 CIRRHOSIS WITHOUT HEPATITIS

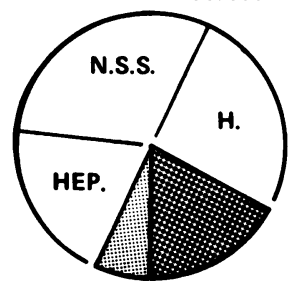

(24\%)
GROUP 2 MILD HEPATITIS

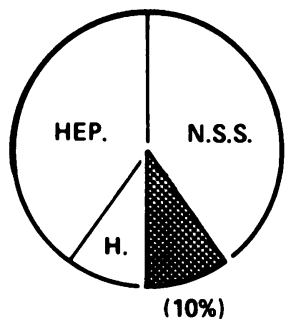

GROUP 5 CIRRHOSIS

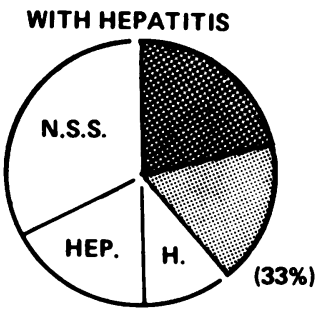

GROUP 3 SEVERE HEPATITIS
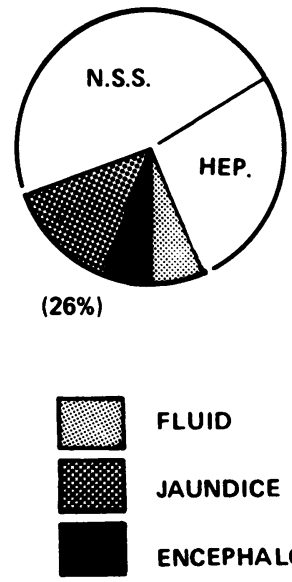

FLUID

JAUNDICE

ENCEPHALOPATHY

HEP. = HEPATOMEGALY

H. = HAEMORRHAGE

N.S.S. $=$ N.S. SYMPTOMS
Fig 1 Mode of presentation (percentages in brackets refer 0 to the proportion of patients presenting with hepatocellular failure-fluid retention, jaun- क्? dice, or encephalopathy).

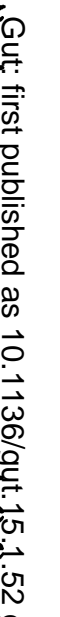




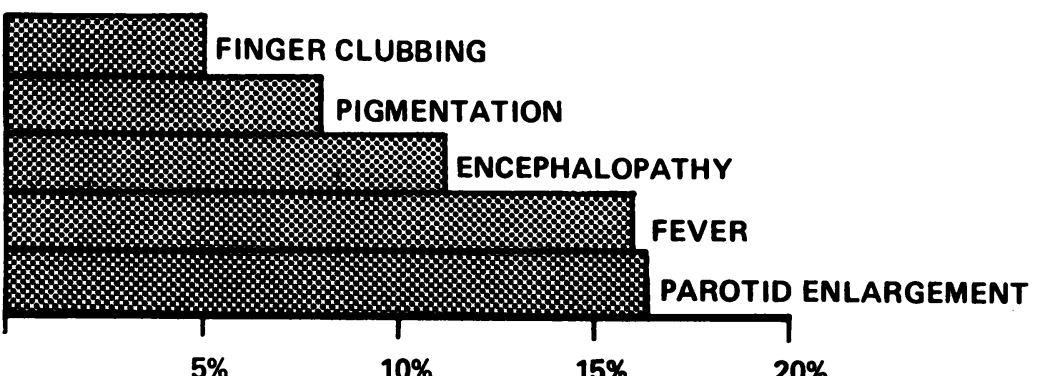

Fig 2 Frequency of some clinical features and associated PANCREATITIS

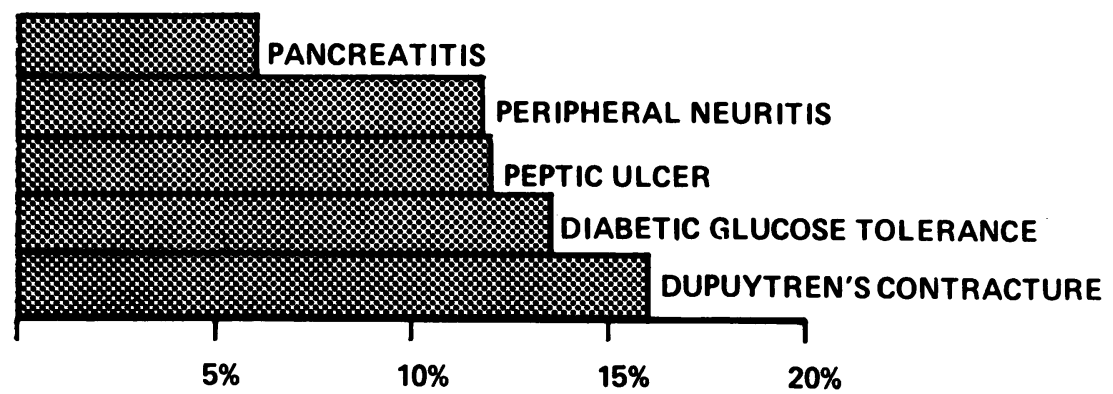
phenomena.

the series. Fever, a feature in $16 \%$ of the series, occurred only in hepatitis and cirrhosis while pigmentation in our experience reflected only cirrhosis. Clubbing was not seen in fatty liver. Dupuytren's contracture, parotid enlargement, and diabetic glucose tolerance were all equally distributed throughout the series regardless of histological subgroup. Peripheral neuropathy was marginally commoner in the cirrhosis subgroup. There were no sex differences.

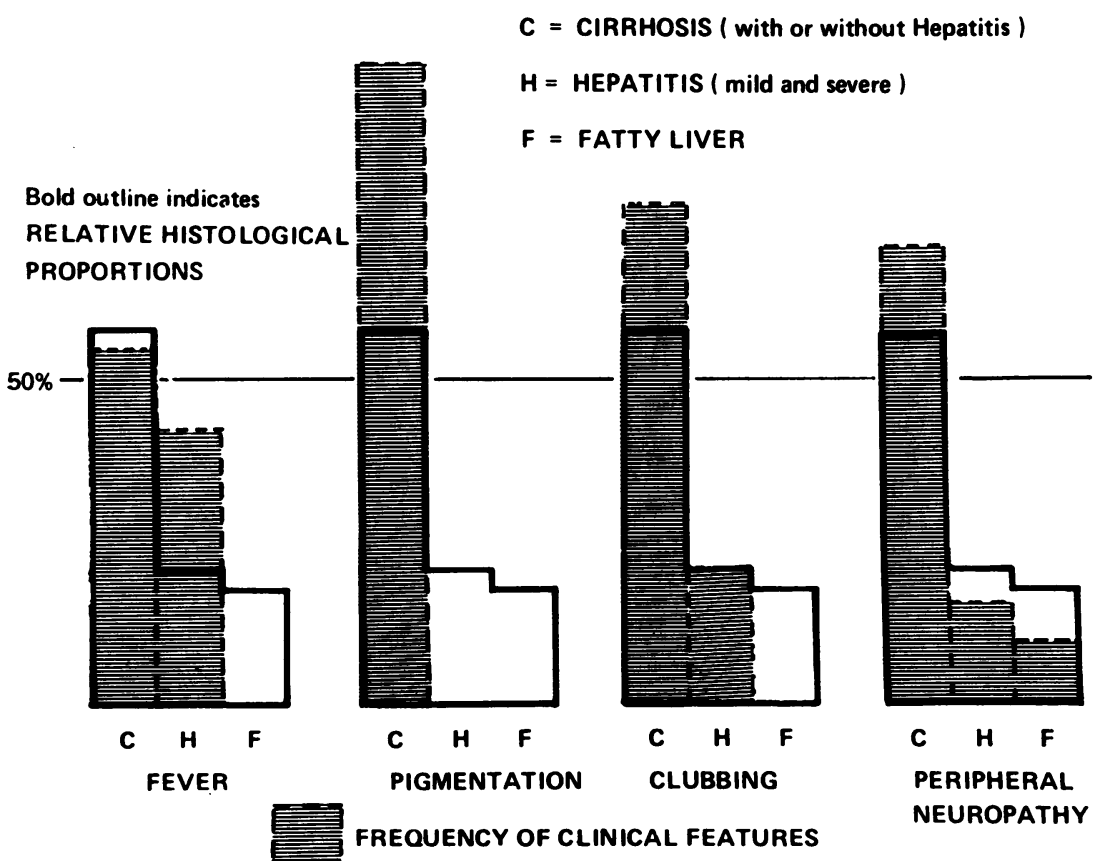

Fig 3 Frequency of some clinical features related to histological subgroup. 

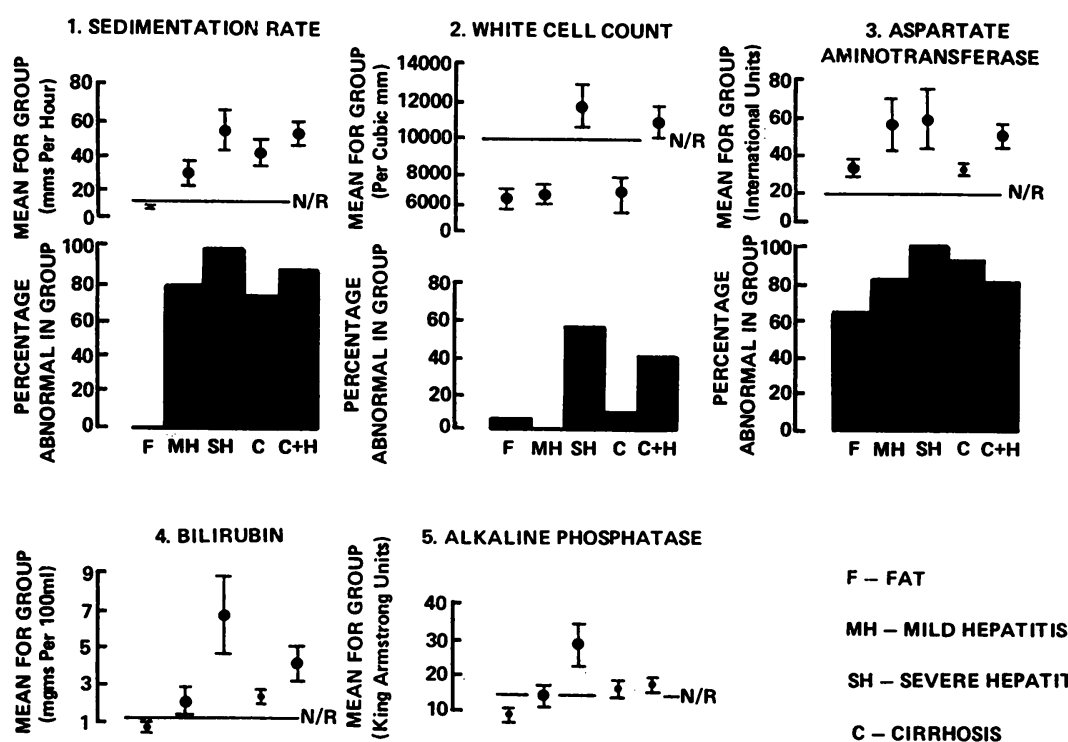

5. ALKALINE PHOSPHATASE
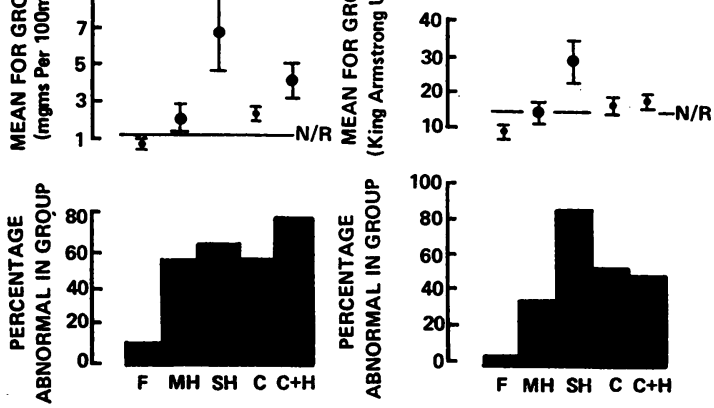

$$
\text { F - FAT }
$$

MH - MILD HEPATITIS

SH - SEVERE HEPATITIS

C - CIRRHOSIS

C+H - CIRRHOSIS WITH HEPATITIS

Fig 4 Laboratory data according to histological subgroup: mean results for group $\vec{i}$ (with standard error) and percentage of group with abnormal results.

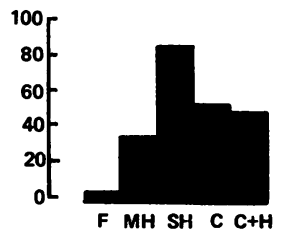

N/R - Normal Range

Cumulative probability of survival

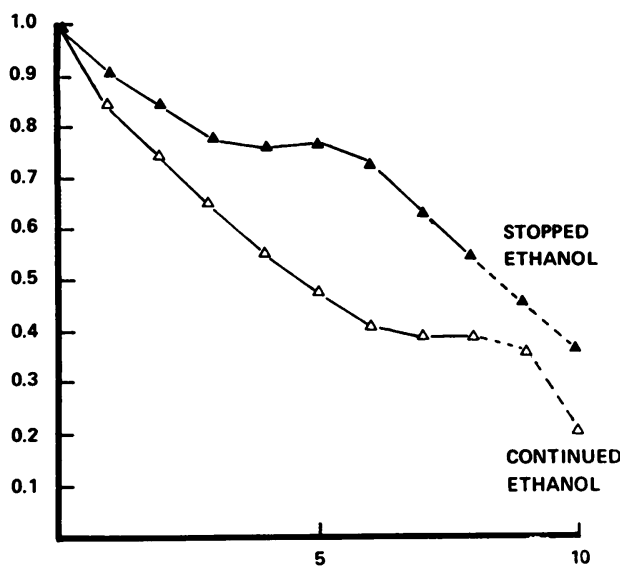

Fig 5a

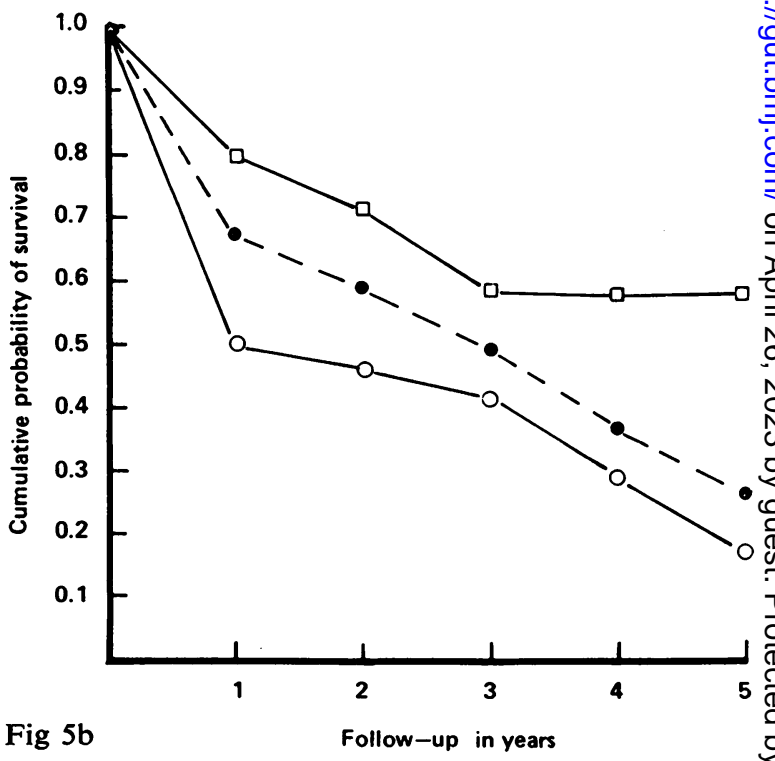

Fig 5 Survivorship curves

A All liver disease according to drinking habits following initial diagnosis

B Cirrhosis compared with hepatitis.

$\square=$ severe hepatitis; $\quad=$ cirrhosis with hepatitis; $\quad$ cirrhosis without hepatitis. 
Figure 4 shows the principal laboratory findings. The most consistent indicators of severe hepatitis or of hepatitis complicating cirrhosis were leucocytosis and hyperbilirubinaemia. The degree of elevation of aminotransferases was less helpful in this respect.

\section{SUR VIVORSHIP}

Mortality in the series was calculated using standard life-table analysis. Figures $5 \mathrm{~A}$ and $\mathrm{B}$ show cumulative probability of survival curves for the entireseries (where drinking habits werecertain, A) and for cirrhosis with and without hepatitis superimposed(B). Fatty liver and mild hepatitis did not contribute significantly to mortality (three deaths in all).

The five-year cumulative probability of survival for cirrhotics continuing alcohol is seen to be $\mathbf{0 . 4 8}$ $(48 \%)$, which contrasts strikingly with that for those abstaining of $0.77(77 \%)$. The mode of death is given in table IV, there being no apparent difference in mode of death between abstainers and nonabstainers. Sex did not appear to play any part in influencing mortality either in length of survival or mode of death.

\begin{tabular}{ll}
\hline Mode of Death & Proportion of Total \\
\hline Coma & $27 \%$ \\
Haemorrhage & $11 \%$ \\
Coma plus haemorrhage & $25 \%$ \\
Renal failure & $13 \%$ \\
Intercurrent infection & $11 \%$ \\
Unrelated causes & $11 \%$ \\
Unknown & $2 \%$ \\
\hline
\end{tabular}

Table IV Mode of death in entire series (258) ${ }^{1}$

${ }^{1}$ Total number of deaths $72(28 \%)$.

\section{Discussion}

It is recognized that needle biopsy of the liver is subject to sampling error. This is greater with macronodular cirrhosis, where regenerative nodules may be sampled, than in the micronodular cirrhosis often found in alcoholics (Scheuer; 1970). We do not believe that such sampling error was a major cause of discrepancy in our findings and in those cases where wedge biopsy or postmortem histology followed closely upon a needle specimen ie, within a few months, the agreement was good. It does, however, make comparison of serial specimens open to error. It is commonly held that cessation of alcohol consumption is followed by a change from micronodular to macronodular cirrhosis (Popper and Schaffner, 1970; Brunt, 1971). This impression could not be confirmed in this study since exactly equal numbers of abstainers and non-abstainers showed an apparent change from micronodular to macronodular picture. But our figures do tend to confirm the impression that alcoholic hepatitis is precirrhotic while fatty change is not. It is also noteworthy that one patient with severe hepatitis who definitely abstained still went on to develop cirrhosis, suggesting that the precirrhotic process may not be reversible by abstention. A similar observation has led Galambos (1972) to suggest that there may be two types of chronic hepatitis in the alcoholic analogous to 'chronic persistent hepatitis' and 'chronic active hepatitis' with a group that may not become cirrhotic despite continuing ethanol consumption and a group developing cirrhosis regardless of abstention. There is recent evidence for altered cellmediated immunity in ethanolic hepatitis which may well contribute to the development or persistence of chronic liver disease (Sorrell and Leevy, 1972).

The centrolobular distribution of the hepatitis lesion was striking and common, being evident in $63 \%$ of biopsies with alcoholic hepatitis with or without cirrhosis. When well developed the centrolobular element has been implicated as the cause of acute portal hypertension and has been termed by Edmondson and his colleagues 'sclerosing hyaline necrosis'(Edmondson, Peters, Reynolds, and Kuzma, 1963). We were not able to show, however, from our series that bleeding from varices was more common in those with sclerosing hyaline necrosis, and, indeed, haemorrhage from any source as a presenting occurrence was not a particularly common feature of hepatitis and was most frequent in cirrhotics without superimposed hepatitis (figure 1). Just over half (58) of those with centrolobular disease also had evidence of cirrhosis. Piecemeal necrosis, albeit usually mild, was present in almost $40 \%$ of cases.

The frequency of apparently severe disease histologically-almost one-third of initial biopsies showed severe hepatitis-was impressive. This 'severity' could not be predicted on clinical presentation alone (figure 1) but did correlate with certain laboratory indices (figure 4). Raised levels of bilirubin and white cell count and to a lesser extent ESR and alkaline phosphatase suggest hepatitis. In that hepatitis, with or without cirrhosis, probably implies a bad prognosis (vide infra) these four indices have some clinical usefulness. The relation of severity of disease to bilirubin and white cell count elevation accords with previous experience. Serum aminotransferases are apparently of little value in predicting histological type or prognosis. Similarly clinical presentation features were not sufficiently discriminatory to be of value in individual cases although fever, pigmentation, and clubbing are pointers to cirrhosis and hepatitis. Since biopsy is sometimes impossible because of disturbance in 
coagulation or lack of available expertise, these clinical features are helpful.

Survival in our series is shown in figures $5 \mathrm{~A}$ and $\mathrm{B}$. In figure 5A survival is shown for the entire group. The overall five-year survival for cirrhosis in the entire series of $54 \%$ accords well with the experience of Powell and Klatskin (1968) but is considerably better than that of the only other British study, that of Stone, Islam, and Paton (1968), whose comparable figure was $20.2 \%$.

The apparently worse survival for cirrhosis without hepatitis (figure 5B) is suprising but the difference is small. Furthermore, mean duration of survival in those cirrhotics with hepatitis was less than those without, being two years four months compared with three years three months respectively, indicating that in general the presence of hepatitis worsens the prognosis. Alcoholic hepatitis carries an appreciable mortality (Hardison and Lee, 1966), although, as our figures suggest (figure 5B), this is largely in the first three years, the survival curve flattening out thereafter.

If the alcoholic cirrhotic stops drinking it is believed to improve his prognosis. From our data (figure 5A) the curves appear to approximate with time approaching 10 years suggesting that abstinence predominantly influences early prognosis. Powell and Klatskin (1968) showed that outlook could be correlated with presentation, being very poor in those with massive haemorrhage and best in those without evidence of jaundice, haematemesis, or ascites. They also showed that there was no significant difference in survival between males and females. In our experience mode of death (coma, renal failure, intercurrent infection, etc) did not differ significantly in the abstainers and the non abstainers.

Poor survival in alcoholic liver disease is associ? ated with continued drinking, the presence of active. hepatitis (suggested by high bilirubin and white celf count), and probably with poor social (and nutri $\mathbb{Q}$ tional) status. Such factors may, however, be moræ important in determining early rather than latg prognosis.

Grateful thanks are expressed to Professor D. F Kerridge of the Department of Statistics, University of Aberdeen, for advice, and to the Department of Medical Illustration, Medical School University of Aberdeen, for figures. This work was supported by the Ingram Trust.

References

Brunt, P. W. (1971). Alcohol and the liver. Gut, 12, 222-229.

Edmondson, H. A., Peters, R. L., Reynolds, T. B., and Kuzma, O. T (1963). Sclerosing hyaline necrosis of the liver in the chronif alcoholic. Ann. intern. Med., 59, 646-673.

Galambos, J. T. (1972). Natural history of alcoholic hepatitis. II Histological changes. Gastroenterology, 63, 1026-1035.

Hardison, W. G., and Lee, F. I. (1966). Prognosis in acute liver diseast of the alcoholic patient. New Engl. J. Med., 275, 61-66.

Office of Health Economics. (1970). Alcohol Abuse (Studies in Curreno Health Problems, 34). O.H.E., London.

Popper, H., Davidson, C. S., Leevy, C. M., and Schaffner, F. (1969) The social impact of liver disease. New Engl. J. Med., 28, 1455-1458

Popper, H., and Schaffner, F. (1970). Structural studies in alcoh and drug induced liver injury. In Alcoholic Cirrhosis and othen Toxic Hepatopathias, edited by A. Engel, and T. Larsso pp. 15-46. Nordiska Bokhandelns, Stockholm.

Powell, W. J., Jr., and Klatskin, G. (1968). Duration of survival in patients with Laennec's cirrhosis. Amer. J. Med., 44, 406-420

Scheuer, P. J. (1970). Liver biopsy in the diagnosis of cirrhosis. Gut: $11,275-278$

Sorrell, M. F., and Leevy, C. M. (1972). Lymphocyte transformation and alcoholic liver injury. Gastroenterology, 63, 1020-1025. वं

Stone, W. D., Islam, N. R. K., and Paton, A. (1968). The natura history of cirrhosis. Quart. J. Med., 145, 119-132. 